Received: 27 April 2018

Accepted: 10 December 2018

Published online: 04 February 2019
NTIFIC REP

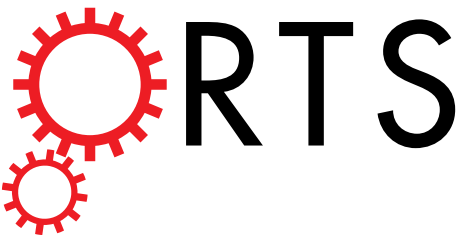

OPEN

\section{Multivariate Control of Effective Cobalt Doping in Tungsten Disulfide for Highly Efficient Hydrogen Evolution Reaction}

\author{
Liyan Zhou ${ }^{1,3}$, Shancheng Yan ${ }^{2}$, Haizeng Song ${ }^{2}$, Han Wu ${ }^{1,3}$ \& Yi Shi ${ }^{1,3}$
}

Tungsten Disulfide $\left(\mathrm{WS}_{2}\right.$ ) is considered to be a promising Hydrogen Evolution Reaction (HER) catalyst to replace noble metals (such as Pt and Pd). However, progress in $\mathrm{WS}_{2}$ research has been impeded by the inertness of the in-plane atoms during HER. Although it is known that microstructure and defects strongly affect the electrocatalytic performance of catalysts, the understanding of such related catalytic origin still remains a challenge. Here, we combined a one-pot synthesis method with wet chemical etching to realize controlled cobalt doping and tunable morphology in $\mathrm{WS}_{2}$. The etched products, which composed of porous $\mathrm{WS}_{2,} \operatorname{CoS}_{2}$ and a spot of $\mathrm{WO}_{x,}$ show a low overpotential and small Tafel slope in $0.5 \mathrm{M} \mathrm{H}_{2} \mathrm{SO}_{4}$ solution. The overpotential could be optimized to $-134 \mathrm{mV}$ (at $10 \mathrm{~mA} / \mathrm{cm}^{2}$ ) with a Tafel slope of $76 \mathrm{mV} / \mathrm{dec}$ at high loadings $\left(5.1 \mathrm{mg} / \mathrm{cm}^{2}\right)$. Under $\mathrm{N}_{2}$ adsorption analysis, the treated $\mathrm{WS}_{2}$ sample shows an increase in macropore $(>50 \mathrm{~nm})$ distributions, which may explain the increase inefficiency of HER activity. We applied electron holography to analyze the catalytic origin and found a low surface electrostatic potential in Co-doped region. This work may provide further understanding of the HER mechanism at the nanometer scale, and open up new avenues for designing catalysts based on other transition metal dichalcogenides for highly efficient HER.

Hydrogen has been considered as a promising clean energy source to remedy the shortage of fossil fuel supply and pollution problems. As such, emerging non-noble catalysts for water splitting are in urgent need. Tungsten Disulfide $\left(\mathrm{WS}_{2}\right)$, one of the two-dimensional transition metal dichalcogenides (TMDCs), has attracted much attention in the catalysis field due to the presence of active sites located along the edges ${ }^{1-4}$, and is expected to replace rare and expensive noble metal catalysts. However, apart from the edges, the majority of the basal surfaces are catalytically inert, which is the main limitation of bare TMDCs compared with noble metal catalysts. The design of the microstructures of such catalyst microstructures thus becomes important, the key directions being the exploration of the influence of pore size distributions, and even electrostatic potential distribution at the surface. There are several approaches reported to overcome the above-mentioned limitation and to enhance the catalytic performance of TMDCs. First, heteroatom doping can significantly modulate the catalytic activity of in-plane TMDC atoms; Deng et al. has systematically reported correlations between dopants and the catalytic activity of in-plane atoms of $2 \mathrm{D} \mathrm{MoS}_{2}{ }^{5}$. Second, multiscale structures may help to promote the liquid-to-gas electrochemical conversion at the interface of catalysts. These mesoporous foams ${ }^{6}$, arrays ${ }^{7,8}$, nanodots ${ }^{9,10}$, and other nanostructures ${ }^{11-13}$ are commonly employed in catalytic researches. Especially for TMDCs, mesopores help to increase the number of edges, thus increasing the number of active sites. Third, high-conducting carbon-based materials including carbon nanotubes ${ }^{14,15}$, graphene ${ }^{16-18}$ and carbon cloth ${ }^{19,20}$ have been mixed with $\mathrm{WS}_{2}$ to improve its electrochemical properties. In addition, $\mathrm{WS}_{2}$ or $\mathrm{MoS}_{2}$ in the metallic $1 \mathrm{~T}$ phase contains a higher density of exposed active sites, better conductivity and catalytic performance ${ }^{21,22}$, which can be obtained by chemical exfoliation from sample in the semiconducting $2 \mathrm{H}_{\text {phase }} \mathrm{e}^{23}$. However, clear understanding of the effect of microstructure design on catalytic performance is still limited.

${ }^{1}$ Collaborative Innovation Center of Advanced Microstructures, Nanjing University, Nanjing, 210093, P. R. China.

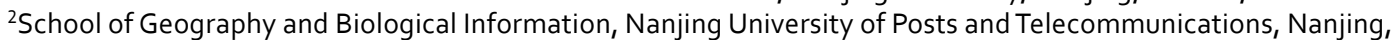
210023, P. R. China. ${ }^{3}$ National Laboratory of Solid State Microstructures, School of Electronic Science and Engineering, Nanjing University, Nanjing, 210093, P. R. China. Correspondence and requests for materials should be addressed to S.Y. (email: yansc@njupt.edu.cn) orY.S. (email: yshi@nju.edu.cn) 

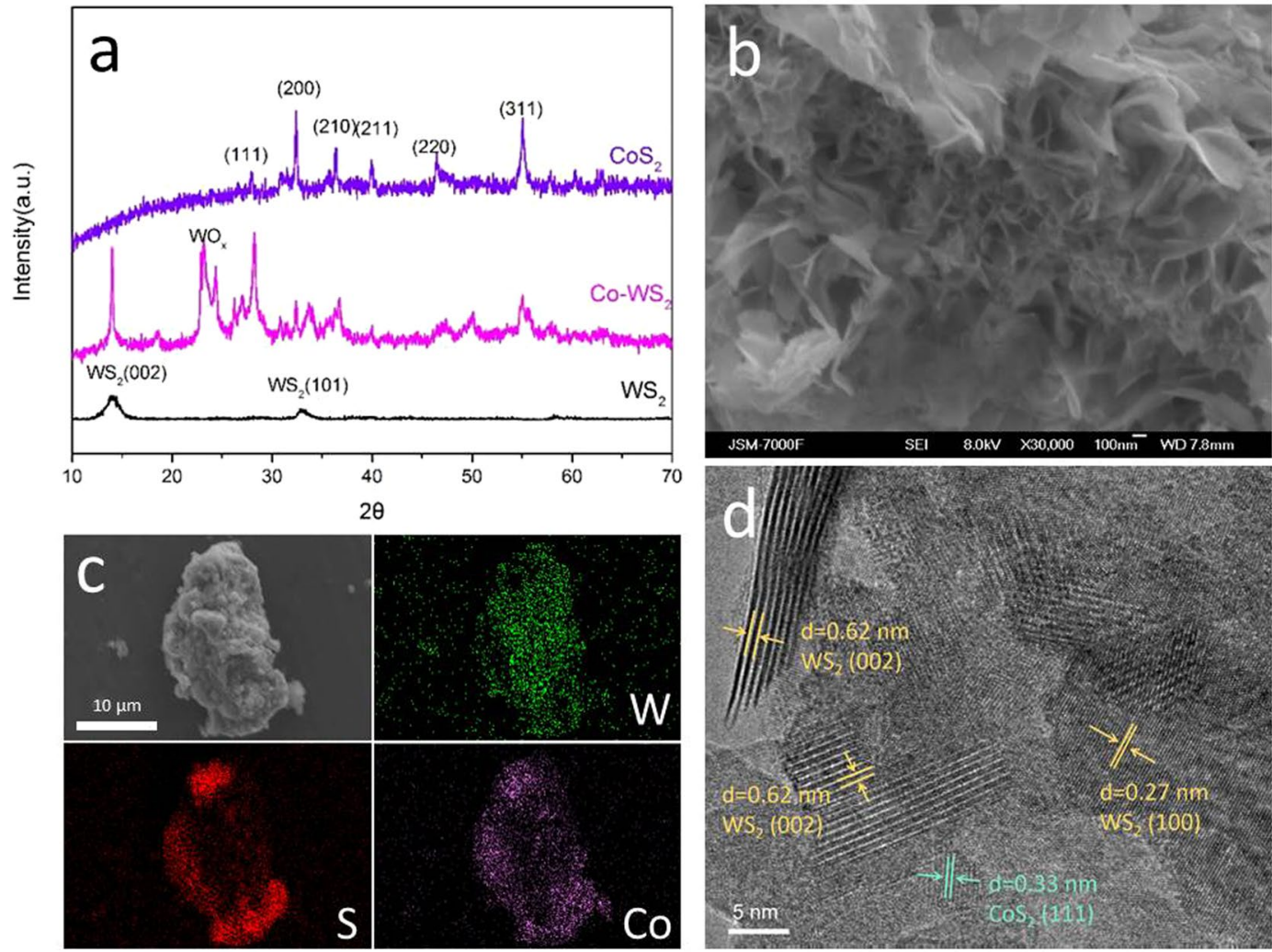

Figure 1. (a) XRD patterns of $\mathrm{WS}_{2}, \mathrm{CoS}_{2}$ and Co-doped $\mathrm{WS}_{2}$, (b) SEM image, (c) Element mapping images from EDS and (d) HRTEM image of Co-doped $\mathrm{WS}_{2}$.

Herein, we combined pore size distribution analysis and electron holography for the first time to solve the above issue. A specific catalyst composed of Cobalt-doped and surface-etched $\mathrm{WS}_{2}$ was synthesized, showing efficient HER activity. We expect that Cobalt-doping will activate the inert in-plane atoms of $\mathrm{WS}_{2}$. Cobalt (Co) is demonstrated to be a good candidate to tune the free energy of hydrogen adsorption of $\mathrm{WS}_{2}$ and some other catalyst candidates ${ }^{19,24}$. Etching by $\mathrm{H}_{2} \mathrm{O}_{2}$ treatment with ultrasonication was applied to modify the morphology of $\mathrm{WS}_{2}{ }^{25}$, and also remove the redundant self-nucleated $\mathrm{CoS}_{2}$. The optimized sample displayed efficient HER performance, with an overpotential of $-134 \mathrm{mV}$ with a small Tafel slope of $76 \mathrm{mV} / \mathrm{dec}$ at a current density of $10 \mathrm{~mA} /$ $\mathrm{cm}^{2}$. The dependence of HER performance on sample microstructures was discussed; the nitrogen absorption test showed a better activity along with increasing macropore $(>50 \mathrm{~nm})$ distributions. An electron hologram has been taken to study the surface electrostatic potential, especially in Co-doped regions, bringing a new viewpoint into HER activity.

\section{Experimental}

Materials. All chemicals used in this work are of analytical grade and applied as received without further purification. Tungsten hexachloride $\left(\mathrm{WCl}_{6}, 99.9 \%\right)$, thioacetamide (TAA, 99\%), N-methylpyrrolidone (NMP, 99\%) and Nafion solution (5\%) were purchased from Sigma-Aldrich. Cobalt nitrate hexahydrate $\left(\mathrm{Co}\left(\mathrm{NO}_{3}\right)_{2} \cdot 6 \mathrm{H}_{2} \mathrm{O}\right.$, 98.5\%), and sulfuric acid $\left(\mathrm{H}_{2} \mathrm{SO}_{4}, 98 \%\right)$ were supplied from Sinopharm Chemical Reagent Co. Ltd. Hydrogen peroxide $\left(\mathrm{H}_{2} \mathrm{O}_{2}, 30 \%\right)$ was purchased from Nanjing Chemical Reagent Co. Ltd.

Synthesis. In a typical synthesis of Co-doped $\mathrm{WS}_{2}\left(\mathrm{Co}-\mathrm{WS}_{2}\right), \mathrm{WCl}_{6}(0.8923 \mathrm{~g}), \mathrm{Co}\left(\mathrm{NO}_{3}\right)_{2} \cdot 6 \mathrm{H}_{2} \mathrm{O}(0.6341 \mathrm{~g})$ and TAA $(1.6904 \mathrm{~g})$ were slowly added to deionized (DI) water $(30 \mathrm{~mL})$ and stirred at room temperature for $1 \mathrm{~h}$. The solution was then transferred to a polyphenylene (PPL) reaction kettle and maintained at $265^{\circ} \mathrm{C}$ for $24 \mathrm{~h}$. The products were cooled to room temperature, centrifuged, washed several times with DI water and ethyl alcohol, and dried at $60^{\circ} \mathrm{C}$. $\mathrm{WS}_{2}$ was synthesized without $\mathrm{Co}\left(\mathrm{NO}_{3}\right)_{2} \cdot 6 \mathrm{H}_{2} \mathrm{O}$ as a control.

For the preparation of $\mathrm{H}_{2} \mathrm{O}_{2}$-treated Co-WS, $100 \mathrm{mg}$ of Co-WS $\mathrm{W}_{2}$ sample was dispersed in $20 \mathrm{~mL}$ of NMP with $2.5 \mathrm{vol} \%$ of $\mathrm{H}_{2} \mathrm{O}_{2}$ and then sonicated for $2 \mathrm{~h}$. The products were centrifuged at $8000 \mathrm{rpm}$, washed several times, and dried at $60^{\circ} \mathrm{C}$. For comparation, Co- $\mathrm{WS}_{2}$ treated by $\mathrm{H}_{2} \mathrm{O}_{2}$ of different concentrations were prepared.

The products were annealed at $450^{\circ} \mathrm{C}$ for $4 \mathrm{~h}$ under an $\mathrm{Ar}$ atmosphere before HER tests. We also studied the influence of different annealing conditions and passivation by 4-nitrobenzene-diazonium (4-NBD) on HER capability and stability, which can be seen in Supplementary Material (Fig. S1).

Characterization. Field emission scanning electron microscopy (FE-SEM; JSM-7000F) was used to investigate the morphology of the samples. Transmission electron microscopy (TEM) and electron holography observations were obtained using a JEOL model JEM2100 instrument at an accelerating voltage of $200 \mathrm{kV}$. Energy 

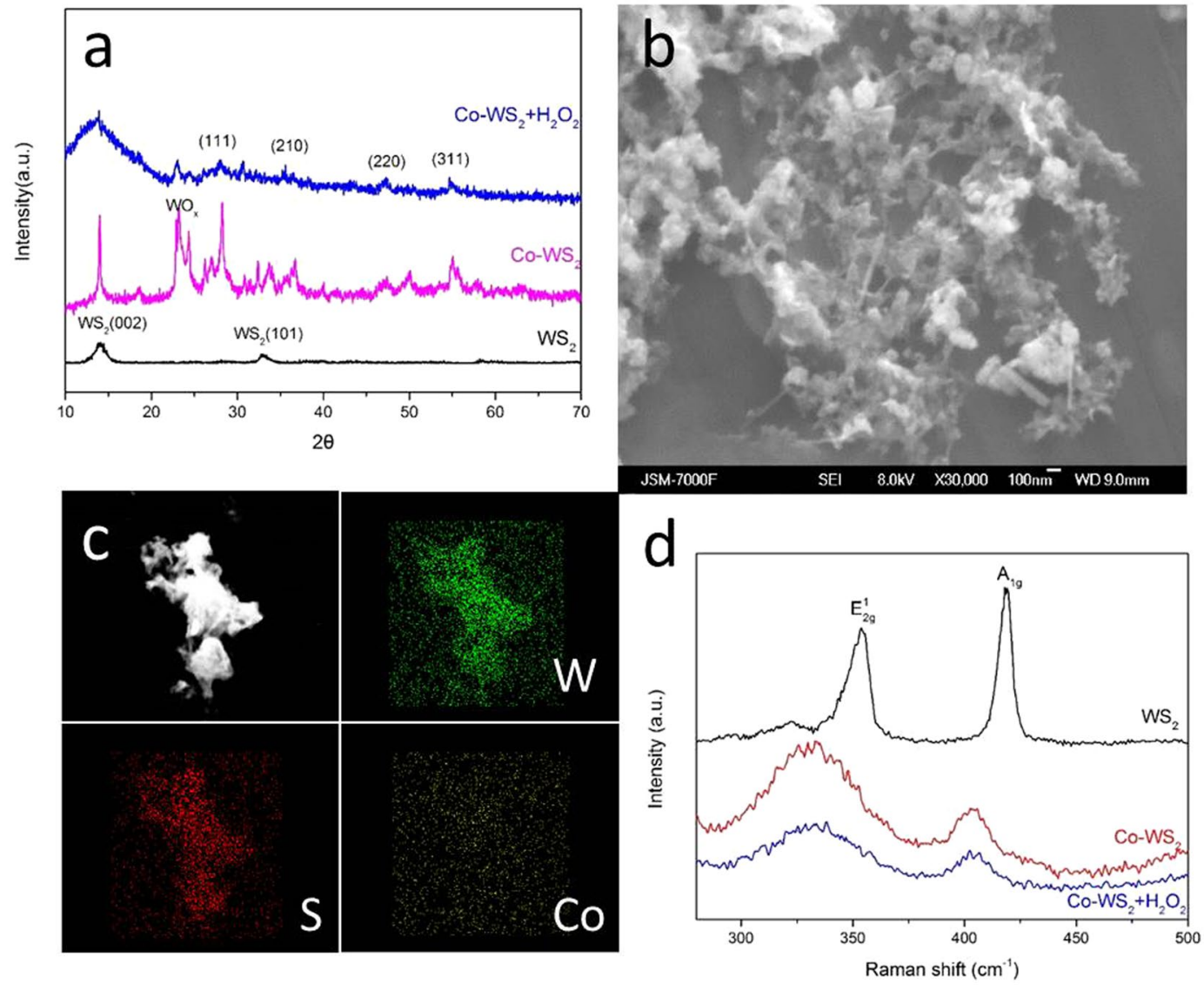

Figure 2. (a) XRD patterns of $\mathrm{WS}_{2}$, Co-doped $\mathrm{WS}_{2}$ and $\mathrm{H}_{2} \mathrm{O}_{2}$ treated Co-WS${ }_{2}$, (b) SEM image and (c) Element mapping images from EDS of $\mathrm{H}_{2} \mathrm{O}_{2}$ treated Co-WS , and (d) Raman spectra of WS $\mathrm{W}_{2}$, Co-doped $\mathrm{WS}_{2}$ and $\mathrm{H}_{2} \mathrm{O}_{2}$ treated Co- $\mathrm{WS}_{2}$.

dispersive spectrometer (EDS; inca X-stream 034A0) was used to confirm the stoichiometry of samples. The crystal phase properties of the samples were analyzed with a Bruker D8 Advance X-ray diffractometer (XRD) using Ni-filtered $\mathrm{Cu} \mathrm{K \alpha}$ radiation at $40 \mathrm{kV}$ and $40 \mathrm{~mA}$ at $2 \theta$ ranging from $10^{\circ}$ to $70^{\circ}$ with a scan rate of $0.02^{\circ}$ per second. Raman spectra were obtained on a Raman spectrometer (LabRam HR800) excited by the $514.5 \mathrm{~nm}$ line of an $\mathrm{Ar}+$ laser under $5 \mathrm{~mW}$. X-ray photoelectron spectroscopy (XPS) analysis (PHI5000 Versaprobe) was used to determine the chemical composition of the products. Brunauer-Emmett-Teller (BET) specific surface area tests were carried out on a Thermo Fisher Surfer at $200^{\circ} \mathrm{C}$.

Electrochemical Measurements. A typical three-electrode set-up was utilized for electrochemical measurement with CHI760D potentiostat (CH Instruments, China). All measurements of the HER activity were conducted using a $0.5 \mathrm{M} \mathrm{H}_{2} \mathrm{SO}_{4}(\mathrm{pH}=0.3)$ electrolyte after continuous purging with $\mathrm{N}_{2}$ gas. A glassy carbon electrode (GCE) with a diameter of $3 \mathrm{~mm}$ covered by a thin catalyst film was used as the working electrode. Typically, $6 \mathrm{mg}$ catalyst was suspended in $1 \mathrm{ml}$ water-ethanol mixed solution (volume ratio of 4:1) containing $20 \mu \mathrm{l}$ Nafion solution to form a homogeneous ink assisted by ultrasonication. Then $20 \mathrm{ml}$ of the ink was dropped onto the surface of glassy carbon by a micropipette and dried under room temperature. Saturated calomel electrode (SCE) and graphite rod ( $\mathrm{Pt}$ anodes may dissolve in the electrolyte and contaminate the cathode) were used as reference electrode and counter electrode respectively. The electrocatalytic activities were examined by polarization curves using linear sweep voltammetry (LSV) at a scan rate of $5 \mathrm{mV} / \mathrm{s}$ with IR compensation, at room temperature. Before measurements, the samples were repeatedly swept from -0.5 to $0 \mathrm{~V}$ (versus SCE) in the electrolyte until a steady voltammogram curve was obtained. Potentials were referenced to a reversible hydrogen electrode (RHE) by adding a value of $0.262 \mathrm{~V}(0.244+0.0591 \times \mathrm{pH})$.

\section{Results and Discussions}

The morphology and chemical composition of as-synthesized Co- $\mathrm{WS}_{2}$ are shown in Fig. 1. The XRD pattern in Fig. 1a shows the differences between Co-doped $\mathrm{WS}_{2}$ and pure $\mathrm{CoS}_{2}$. The diffraction peaks of both pure $\mathrm{CoS}_{2}$ and Co-doped $\mathrm{WS}_{2}$ correspond to $\mathrm{CoS}_{2}$ (JCPDS card no. 41-1471). However, the doped Co has a stronger (111) diffraction $\left(27.9^{\circ}\right)$, while pure $\mathrm{CoS}_{2}$ has stronger (200) and (311) diffractions, corresponding to peaks at $32.3^{\circ}$ and $54.9^{\circ}$ respectively. There are two peaks at $14.4^{\circ}$ and $33.6^{\circ}$, assigned to diffractions of the (002) and (101) planes of $\mathrm{WS}_{2}$ (JCPDS card no. 08-0237) respectively. It is worth mentioning that the Co-doping also oxidizes $\mathrm{WS}_{2}$, with observed diffraction peaks at around $25^{\circ 26}$. Though the mechanism of oxidation is still under investigation, 

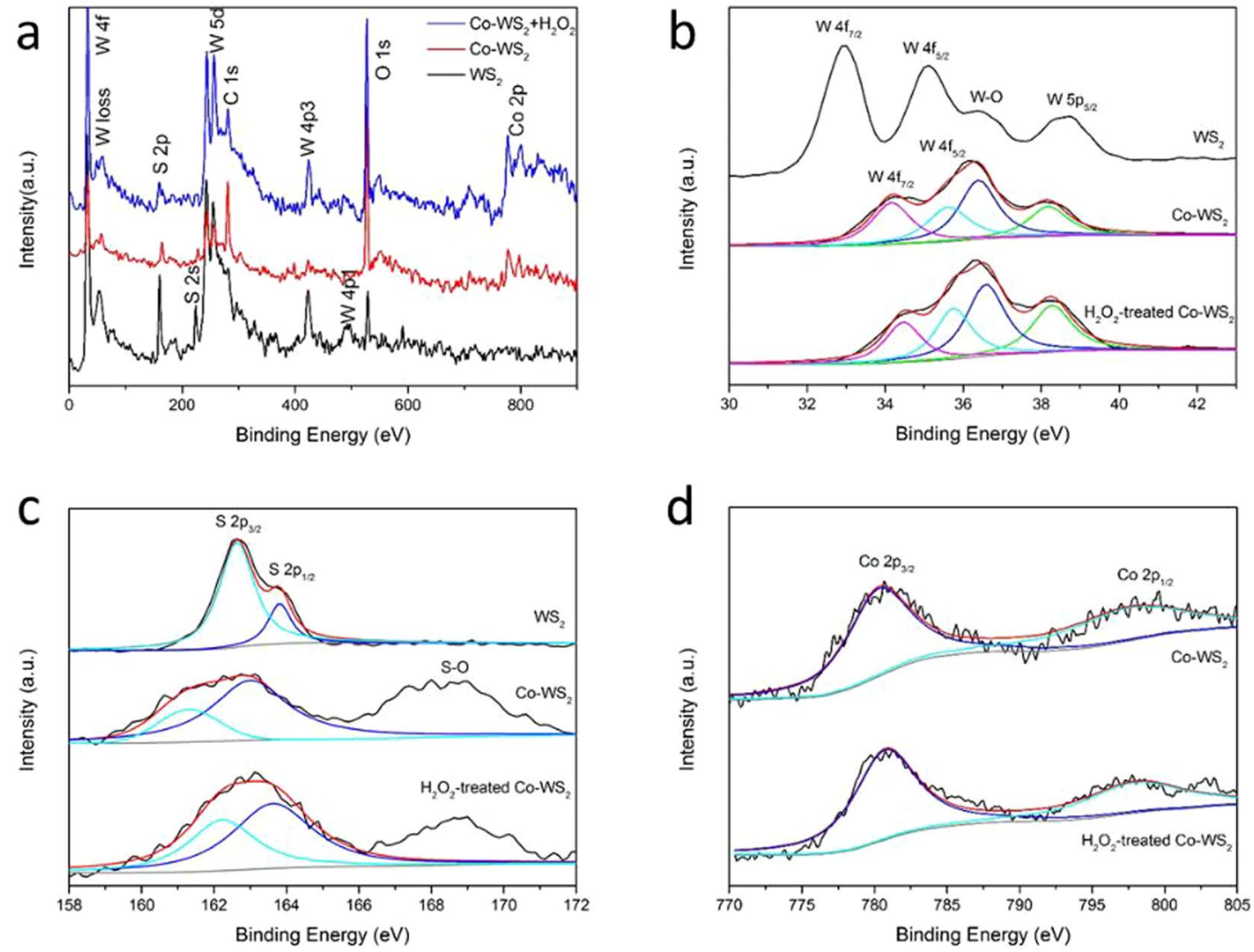

Figure 3. XPS spectra of $\mathrm{WS}_{2}$, Co-doped $\mathrm{WS}_{2}$ and $\mathrm{H}_{2} \mathrm{O}_{2}$ treated Co- $\mathrm{WS}_{2}$. (a) Survey scan, (b) W 4f, (c) S 2p and (d) Co 2 p peaks, respectively.

tungsten oxides $\left(\mathrm{WO}_{\mathrm{x}}\right)$ have higher electrical conductivities than the metallic phase $1 \mathrm{~T}-\mathrm{WS}_{2}$, and may bring additional benefits to HER ${ }^{27-29}$. The typical three-dimensional nanosheet structures of $\mathrm{WS}_{2}$ can be observed in SEM (Fig. 1b), while massive self-nucleated $\mathrm{CoS}_{2}$ also exists (Fig. S2). EDS mapping (Fig. 1c) indicates a homogeneous distribution of Co element, instead of dissociative $\mathrm{CoS}_{2}$ shown in Fig. S2. Figure 1d shows a HRTEM image of $\mathrm{Co}-\mathrm{WS}_{2}$, detailing the crystal structure of the product. The lattice plane (002) of $\mathrm{WS}_{2}$ with a spacing distance of about $6.2 \AA$ can be observed, as well as the lattice plane (100) of $\mathrm{WS}_{2}$ with a shorter spacing distance of $2.7 \AA$. Another plane of middle spacing distance of $3.3 \AA$ can be also found, which corresponds to the lattice plane (111) of $\mathrm{CoS}_{2}$, which corroborates with the XRD results.

After etching of $\mathrm{WS}_{2}$ by $\mathrm{H}_{2} \mathrm{O}_{2}$, the intensity of $\mathrm{CoS}_{2}$ peaks decreased (Fig. 2a), corresponding to the notable decrease of Co content found in EDS mapping (Fig. 2c). However, the (002) diffraction peak of $\mathrm{WS}_{2}$ became broader, which is caused by poor crystallinity. The Co-WS $\mathrm{W}_{2}$ samples became smaller after $\mathrm{H}_{2} \mathrm{O}_{2}$ etching and ultrasonication, while pieces of $\mathrm{WS}_{2}$ were still observed as displayed in Fig. 2b. Such a porous nanosheet structure is propitious for HER. Raman spectroscopy was conducted for $\mathrm{WS}_{2}, \mathrm{Co}-\mathrm{WS}_{2}$ and $\mathrm{H}_{2} \mathrm{O}_{2}$ treated Co-WS . As shown in Fig. $2 \mathrm{~d}$, the peaks observed at $353 \mathrm{~cm}^{-1}$ and $418 \mathrm{~cm}^{-1}$ correspond to the $\mathrm{E}_{2 \mathrm{~g}}^{1}$ and $\mathrm{A}_{1 \mathrm{~g}}$ modes of $\mathrm{WS}_{2}$, respectively. $\mathrm{E}_{2 \mathrm{~g}}^{1}$ is an in-plane optical mode, while $\mathrm{A}_{1 \mathrm{~g}}$ corresponds to out-of plane vibrations of the sulfur atoms ${ }^{30,31}$. However, both the two bands of Co- $\mathrm{WS}_{2}$ and $\mathrm{H}_{2} \mathrm{O}_{2}$ treated $\mathrm{Co}-\mathrm{WS}_{2}$ show an obvious shift to $330 \mathrm{~cm}^{-1}$ and $404 \mathrm{~cm}^{-1}$ respectively, as compared to pure $\mathrm{WS}_{2}$. This change of red shift should be ascribed to the influence of small amounts of doping to the host materials ${ }^{32}$.

$\mathrm{X}$-ray photoelectron spectroscopy (XPS) spectra are shown in Fig. 3 to confirm the elemental composition of the products. Figure 3a shows the typical survey spectrum of $\mathrm{WS}_{2}, \mathrm{Co}$-doped $\mathrm{WS}_{2}$ and $\mathrm{H}_{2} \mathrm{O}_{2}$ treated Co-WS . Here, the presence of C 1s, W 4f, S 2p, and O 1 s regions may be observed in both samples. Specifically, Co $2 \mathrm{p}$ regions can be seen in Co-doped samples. In Fig. 3b, compared to bare $\mathrm{WS}_{2}$, two peaks of $4 \mathrm{f}$-level W atoms shift to higher binding energy, which is probably caused by Co-doping. It is found that the $\mathrm{W}$-O bond at $36.5 \mathrm{eV}$ shows a relative increase in Co-doping samples, demonstrating oxidation along with Co-doping. Meanwhile, the conversion of $\mathrm{W}$ valence state from $+4\left(\mathrm{WS}_{2}\right)$ to $+6\left(\mathrm{WO}_{3}\right)$ could also result in the increase of binding energy ${ }^{26}$. In Fig. $3 c$, the $S 2 p_{3 / 2}$ and $2 p_{1 / 2}$ peaks at 162.6 and $163.8 \mathrm{eV}$ suggest the -2 valence state for $S$. Co-doping leads to the formation of S-O bond, shown as the peak at around $169 \mathrm{eV}$. This decreases the binding energy for S $2 \mathrm{p}$ bonds. Two peaks shown in Fig. $3 \mathrm{~d}$ at binding energy of 780.6 and $798.0 \mathrm{eV}$ corresponding to $\mathrm{Co} 2 \mathrm{p}_{3 / 2}$ and $2 \mathrm{p}_{1 / 2}$, are almost consistent with the results reported for $\mathrm{CoS}_{2}{ }^{33}$.

We tested the electrocatalytic HER performance using a simple three-electrode set-up in $0.5 \mathrm{M} \mathrm{H}_{2} \mathrm{SO}_{4}$ solution. Figure 4 a shows the polarization curves measured by Linear-sweep voltammetry (LSV) at a scan rate $0.5 \mathrm{mV} / \mathrm{s}$. Bare $\mathrm{WS}_{2}$ and bare $\mathrm{CoS}_{2}$ both showed poor HER performances, and $\mathrm{CoS}_{2}$ even showed a reduction peak during cathodic polarization that disappeared after hundreds of scans. The Co-doped $\mathrm{WS}_{2}$ sample showed a 

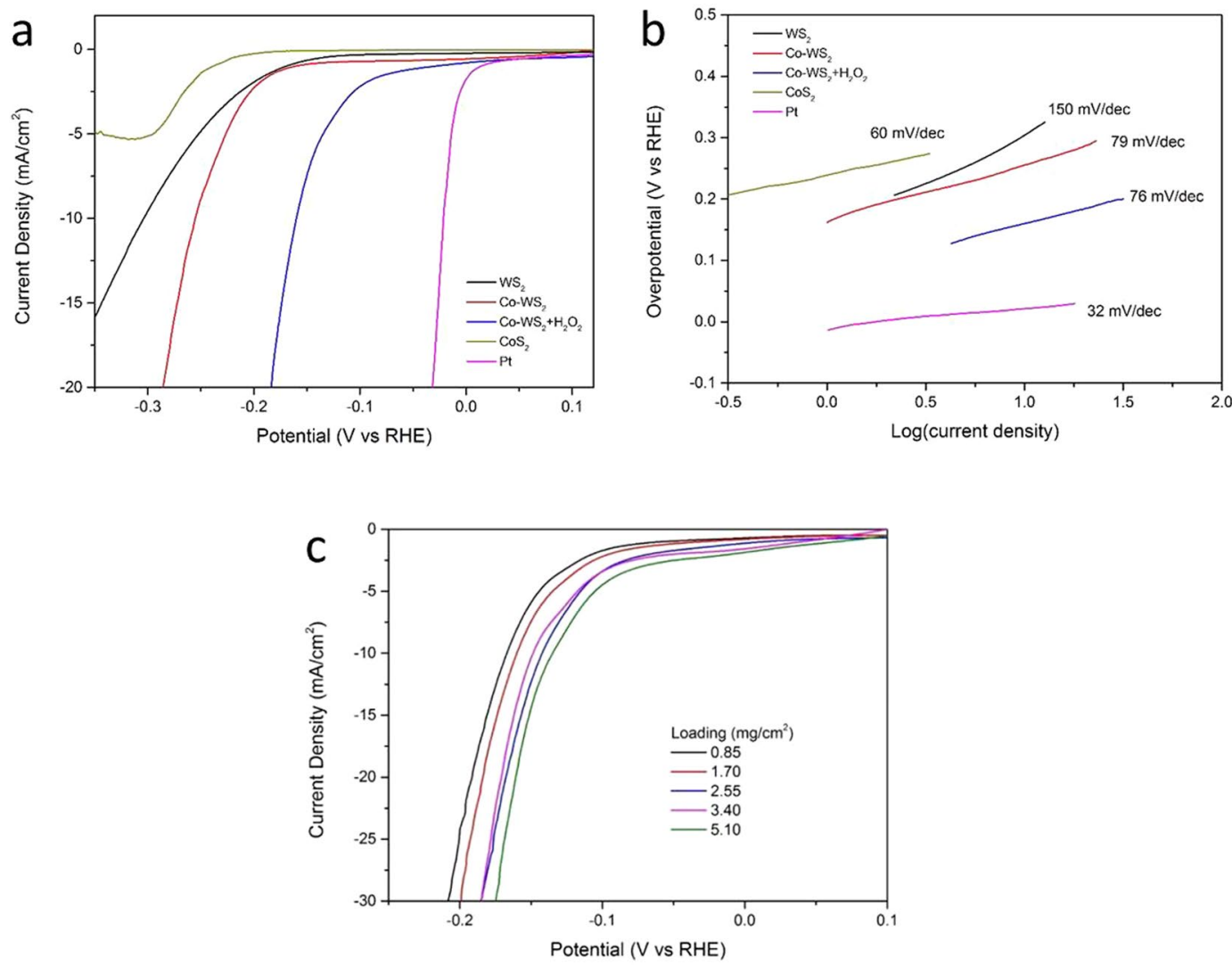

Figure 4. Effect of Co doping and $\mathrm{H}_{2} \mathrm{O}_{2}$ treatment on the HER (a) polarization curves and (b) corresponding Tafel plots of a series of samples loading of $1.7 \mathrm{mg} / \mathrm{cm}^{2}$. (c) Polarization curves recorded with different amount loading.

a

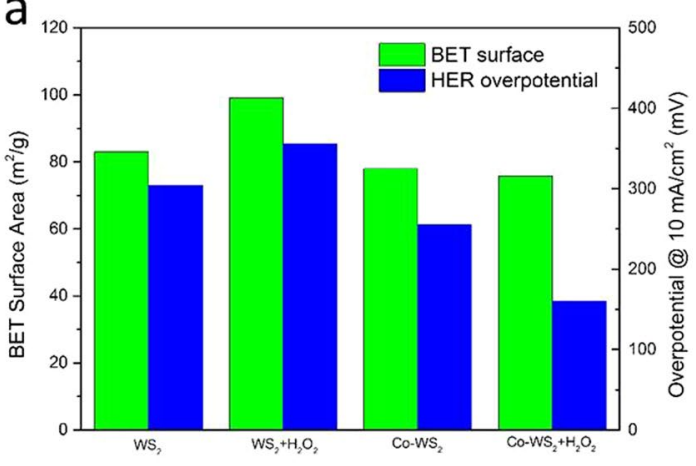

b

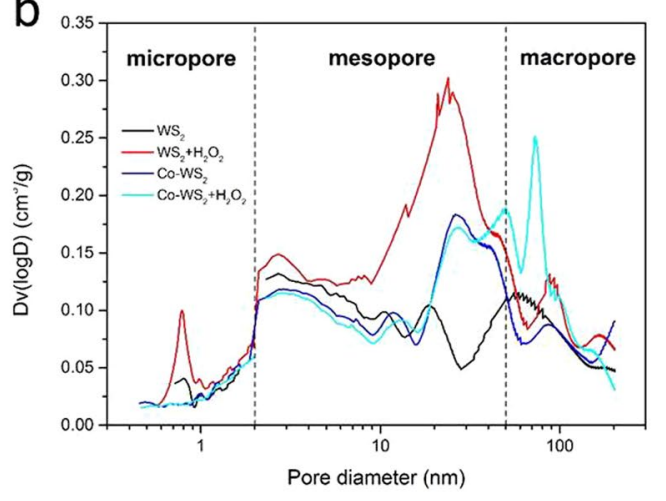

Figure 5. (a) Histogram of measured BET surface areas and HER overpotentials (at $\left.10 \mathrm{~mA} / \mathrm{cm}^{2}\right)$, and (b) Pore size distributions based on $\mathrm{BJH}$ method of serial samples.

good HER performance with an overpotential of $-255 \mathrm{mV}$ at the current density of $-10 \mathrm{~mA} / \mathrm{cm}^{2}$. The influence of Co-doping with different amounts has been discussed, as shown in Fig. S3. Considering the self-nucleation of $\mathrm{CoS}_{2}$ at the surface of $\mathrm{WS}_{2}$, we have tried another method to realize Co-doping. The results are still under study, as displayed in Fig. S4.

The $\mathrm{H}_{2} \mathrm{O}_{2}$ treated Co- $\mathrm{WS}_{2}$ reached a much smaller overpotential of $-160 \mathrm{mV}$ at $-10 \mathrm{~mA} / \mathrm{cm}^{2}$, showing a better HER performance that approaches that of the commercial $20 \% \mathrm{Pt} / \mathrm{C}$ catalyst $\left(-21 \mathrm{mV} @-10 \mathrm{~mA} / \mathrm{cm}^{2}\right)$. By fitting linear regions of the LSV plots to the Tafel equation $\eta=a+b \log j$, where $j$ is the current density and $\mathrm{b}$ is the Tafel slope ${ }^{34}$, the corresponding Tafel plots of each sample were shown in Fig. 4b. Tafel slopes of $20 \%$ $\mathrm{Pt} / \mathrm{C}, \mathrm{H}_{2} \mathrm{O}_{2}$-treated $\mathrm{Co}-\mathrm{WS}_{2}, \mathrm{Co}-\mathrm{WS}_{2}$, bare $\mathrm{CoS}_{2}$ and bare $\mathrm{WS}_{2}$ were extracted as $32,76,79,60$, and $150 \mathrm{mV} / \mathrm{dec}$, respectively. The Co-doped $\mathrm{WS}_{2}$ samples showed smaller Tafel slopes than that of $\mathrm{WS}_{2}$, indicating a faster gain of HER velocity with increasing potential. Also, even though the HER activity of $\mathrm{CoS}_{2}$ is relatively low, the Tafel 

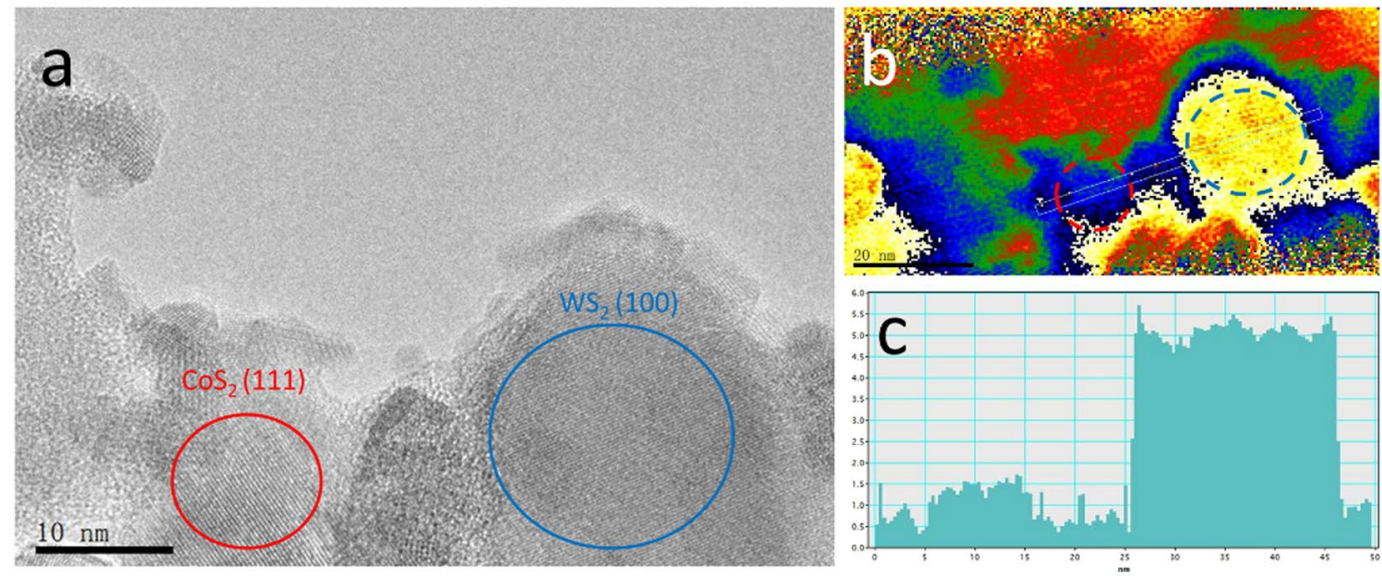

Figure 6. Electron holographic analysis: (a) The TEM image showing Co-doping nearby $\mathrm{WS}_{2}$, (b) phase reconstructed of the hologram corresponding to $(\mathbf{a}, \mathbf{c})$ phase profile measured along the strip in (b).

slope is similar to that of a Pt/C catalyst, which may reflect the effect of Co doping. Electrochemical impedance spectroscopy (EIS) was used to investigate the electrode kinetics under the HER test conditions, confirming the facile kinetics of $\mathrm{H}_{2} \mathrm{O}_{2}$-treated Co-WS toward HER (Fig. S5).

We then investigated the HER performance of the best sample $\left(\mathrm{H}_{2} \mathrm{O}_{2}\right.$ treated Co- $\left.\mathrm{WS}_{2}\right)$ under different loadings, as shown in Fig. 4c. It was found that the catalytic performance got even better with heavier loading, and the overpotential could reach $-134 \mathrm{mV}$ when the loading on GCE was $5.1 \mathrm{mg} / \mathrm{cm}^{2}$. However, when the loading was further increased (by increasing the concentration of dispersion ink or enhancing the dropped amount on GCE), the dry film of catalyst on GCE became fragile and fell off easily, causing poor electrical conductivity and HER stability. Durability measurement of $\mathrm{H}_{2} \mathrm{O}_{2}$-treated $\mathrm{Co}-\mathrm{WS}_{2}$ was carried out with a small loading of $0.85 \mathrm{mg} /$ $\mathrm{cm}^{2}$ (Fig. S6).

Characterizations indicated that the Co-doping caused oxidation in the final products. Compared to bare $\mathrm{WS}_{2}$, Co-doped WS $\mathrm{W}_{2}$ consists of W, Co, S, and $\mathrm{O}$ elements. We applied $\mathrm{H}_{2} \mathrm{O}_{2}$ in order to etch the surface of $\mathrm{WS}_{2}$, but also caused a massive loss of Co. Considering the better HER performance of $\mathrm{H}_{2} \mathrm{O}_{2}$ treated Co-WS , we speculate that appropriate $\mathrm{H}_{2} \mathrm{O}_{2}$ etching could remove redundant self-nucleated $\mathrm{CoS}_{2}$ covering or mixed with $\mathrm{WS}_{2}$, which possesses poor HER activity. The remaining Co content plays a role in tuning the free energy of hydrogen adsorption of $\mathrm{WS}_{2}$ atoms, especially inert in-plane atoms. The above-mentioned lead to a highly efficient HER performance, manifested as good electrical conductivities of tungsten oxide $\left(\mathrm{WO}_{\mathrm{x}}\right)$.

In order to quantitatively analyze the dependence of HER performance on sample microstructures, we carried out independent $\mathrm{N}_{2}$ adsorption measurements based on Brunauer-Emmett-Teller (BET) models on these powder samples. The BET surface area and HER overpotential (at $10 \mathrm{~mA} / \mathrm{cm}^{2}$ ) of each sample were compared in Fig. 5a. Based on our understanding, bigger surface areas of catalyst should be associated with more active sites. Especially for $\mathrm{WS}_{2}$, the mesopores may increase the number of edges as the active sites and increase the accessibility of the catalyst surface. That is the reason why we chose $\mathrm{H}_{2} \mathrm{O}_{2}$ to etch $\mathrm{WS}_{2}$ into ultrathin and porous nanosheets. The BET measurement confirmed the influences of etching on $\mathrm{WS}_{2}$, and the surface area of $\mathrm{WS}_{2}$ was enhanced from 82.9 to $99.0 \mathrm{~m}^{2} / \mathrm{g}$. The BET surface area of Co-doped $\mathrm{WS}_{2}$ is slightly smaller than that of $\mathrm{WS}_{2}$, due to the Co-doping into the interspace of $\mathrm{WS}_{2}$ and the self-nucleation of $\mathrm{CoS}_{2}$. However, the relation between HER performance and surface area of $\mathrm{WS}_{2}$ samples is unexpected. The higher surface area of $\mathrm{H}_{2} \mathrm{O}_{2}$-treated WS $\mathrm{S}_{2}$ led to a worse HER performance than that of bare $\mathrm{WS}_{2}$, and the lowest surface area of $\mathrm{H}_{2} \mathrm{O}_{2}$-treated Co- $\mathrm{WS}_{2}$ corresponds to the best HER efficiency. For better understanding, pore size distributions were calculated from adsorption branches of isotherms by the Barrett-Joyner-Halenda (BJH) method, as shown in Fig. 5b. The detailed pore size distributions suggest that serial $\mathrm{WS}_{2}$ samples contain a mixture of macropores $(>50 \mathrm{~nm})$, mesopores $(2-50 \mathrm{~nm})$, and a small amount of micropores $(<2 \mathrm{~nm})$. Compared to bare $\mathrm{WS}_{2}$, the $\mathrm{H}_{2} \mathrm{O}_{2}$ etching caused an increase in mesopore distribution ranging from 10 to $50 \mathrm{~nm}$, as well as micropores of $0.8 \mathrm{~nm}$ and macropores of $90 \mathrm{~nm}$. The increased mesopores caused a larger BET surface area, but had no impact on the HER. The Co-doped $\mathrm{WS}_{2}$ also possesses a broad mesopore distribution, which is probably contributed by $\mathrm{CoS}_{2}$. The subsequent $\mathrm{H}_{2} \mathrm{O}_{2}$ treatment resulted in macropore distributions at $50 \mathrm{~nm}$ and $73 \mathrm{~nm}$. The influence of $\mathrm{H}_{2} \mathrm{O}_{2}$ concentrations is discussed in supplementary materials (Fig. S7). Considering the above results, we speculate that both Co doping and $\mathrm{H}_{2} \mathrm{O}_{2}$ etching almost could not influence the pore $(<10 \mathrm{~nm})$ distribution of $\mathrm{WS}_{2}$. Thus the macropores etched by $\mathrm{H}_{2} \mathrm{O}_{2}$ associated with Co-doping may account for the enhanced HER performance.

To understand the effect of doped Co content in the improvement of HER activity of $\mathrm{WS}_{2}$, electron holographic imaging has been taken to study the inner electrostatic potential of our samples. The amount of phase shift can provide a direct measure of the variation in electrostatic potential at the surface of nonmagnetic specimens with uniform thickness ${ }^{35,36}$. We selected a thin specimen containing $\mathrm{CoS}_{2}$ and $\mathrm{WS}_{2}$, as shown in Fig. 6(a). The corresponding hologram was taken using a transmission electron microscope equipped with an electron biprism and processed by two dimensional Fourier transformation to obtain the side-bands. The phase was reconstructed from the side-bands by inverse Fourier transformation, shown in Fig. 6(b). Figure 6(c) displayed the potential step along the dashed box in Fig. 6(b), showing a lower inner electrostatic potential in Co-doped 
regions of the sample. The Gibbs free energy of $\mathrm{WS}_{2}$ or $\mathrm{MoS}_{2}$ is too strong, leading to the poisoning of the active sites, especially in inner planes. Electronic states of in plane atoms would be significantly modulated by Co doping, thus leading to a moderate hydrogen adsorption free energy with a Gibbs free energy close to 0 for highly efficient HER ${ }^{6,37}$. The hologram may explain the modulation of electronic states. This is the first use of electrostatic potential measured by hologram to distinguish the activity in a single region of catalysts. DFT calculation is needed for further insights.

\section{Conclusions}

In summary, we studied the influence of microstructure on high-performance Co-doped $\mathrm{WS}_{2}$-based HER catalyst. $\mathrm{H}_{2} \mathrm{O}_{2}$ etching was used to remove redundant self-nucleated $\mathrm{CoS}_{2}$ and modify the surface morphology of $\mathrm{WS}_{2}$, thus creating macropores. Various characterization results indicated that the products were composed of $\mathrm{WS}_{2}, \mathrm{CoS}_{2}$ and a spot of $\mathrm{WO}_{\mathrm{x}}$, showing a low HER overpotential of $-134 \mathrm{mV}\left(10 \mathrm{~mA} / \mathrm{cm}^{2}\right.$ at a loading of $5.1 \mathrm{mg} /$ $\mathrm{cm}^{2}$ ), with a small Tafel slope of $76 \mathrm{mV} /$ dec. Co-doping associated with $\mathrm{H}_{2} \mathrm{O}_{2}$ resulted in an increasing macropore $(>50 \mathrm{~nm})$ distributions, which may account for the enhanced HER performance. For the first time, we used electron holographic analysis to study the electrostatic potential in Co-doped region. Further work is required, such as carbon material hybridization and DFT calculation, and we believe our study could bring in better understanding of promising catalysts design to replace noble metal for hydrogen production.

\section{References}

1. Jaramillo, T. F. et al. Identification of active edge sites for electrochemical $\mathrm{H}_{2}$ evolution from $\mathrm{MoS}_{2}$ nanocatalysts. Science 317 , 100-102 (2007)

2. Lukowski, M. A. et al. Highly active hydrogen evolution catalysis from metallic $\mathrm{WS}_{2}$ nanosheets. Energy Environ. Sci. 7, 2608-2613 (2014).

3. Gholamvand, Z., McAteer, D., Harvey, A., Backes, C. \& Coleman, J. N. Electrochemical applications of two-dimensional nanosheets: The effect of nanosheet length and thickness. Chem. Mater. 28, 2641-2651 (2016).

4. Yin, Y. et al. Contributions of phase, sulfur vacancies, and edges to the hydrogen evolution reaction catalytic activity of porous molybdenum disulfide nanosheets. J. Am. Chem. Soc. 138, 7965-7972 (2016).

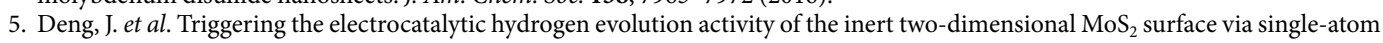
metal doping. Energy Environ. Sci. 8, 1594-1601 (2015).

6. Deng, J. et al. Multiscale structural and electronic control of molybdenum disulfide foam for highly efficient hydrogen production. Nat. Commun. 8, 14430 (2017).

7. Peng, S. et al. Engineering $\mathrm{Co}_{9} \mathrm{~S}_{8} / \mathrm{WS}_{2}$ array films as bifunctional electrocatalysts for efficient water splitting. J. Mater. Chem. A 5, 23361-23368 (2017).

8. Yang, H., Zhang, Y., Hu, F. \& Wang, Q. Urchin-like CoP nanocrystals as hydrogen evolution reaction and oxygen reduction reaction dual-electrocatalyst with superior stability. Nano Lett. 15, 7616-7620 (2015).

9. Tan, C. et al. Preparation of High-Percentage 1T-Phase Transition Metal Dichalcogenide Nanodots for Electrochemical Hydrogen Evolution. Adv. Mater. 30, 1705509 (2018).

10. Xu, S., Li, D. \& Wu, P. One-pot, facile, and versatile synthesis of monolayer $\mathrm{MoS}_{2} / \mathrm{WS}_{2}$ quantum dots as bioimaging probes and efficient electrocatalysts for hydrogen evolution reaction. Adv. Funct. Mater. 25, 1127-1136 (2015).

11. Xie, J. et al. Intralayered Ostwald Ripening to Ultrathin Nanomesh Catalyst with Robust Oxygen-Evolving Performance. Adv. Mater. 29, 1604765 (2017).

12. Li, H. et al. Atomic-Sized Pores Enhanced Electrocatalysis of $\mathrm{TaS}_{2}$ Nanosheets for Hydrogen Evolution. Adv. Mater. 28, 8945-8949 (2016).

13. Wu, A. et al. Hierarchical $\mathrm{MoS}_{2} @ \mathrm{MoP}$ core-shell heterojunction electrocatalysts for efficient hydrogen evolution reaction over a broad pH range. Nanoscale 8, 11052-11059 (2016).

14. Yu, S. et al. Rational design of efficient electrocatalysts for hydrogen evolution reaction: single layers of WS $\mathrm{S}_{2}$ nanoplates anchored to hollow nitrogen-doped carbon nanofibers. ACS Appl. Mater. Inter. 7, 28116-28121 (2015).

15. He, Q. et al. In situ growth of metallic $1 \mathrm{~T}-\mathrm{WS}_{2}$ nanoislands on single-walled carbon nanotube films for improved electrochemical performance. RSC Adv. 6, 87919-87925 (2016).

16. Yang, J. et al. Two-dimensional hybrid nanosheets of tungsten disulfide and reduced graphene oxide as catalysts for enhanced hydrogen evolution. Angew. Chem. Int. Ed. 52, 13751-13754 (2013).

17. Luxa, J., Fawdon, J., Sofer, Z., Mazánek, V. \& Pumera, M. $\mathrm{MoS}_{2} / \mathrm{WS}_{2}$-Graphene Composites through Thermal Decomposition of Tetrathiomolybdate/Tetrathiotungstate for Proton/Oxygen Electroreduction. Chemphyschem 17, 2890-2896 (2016).

18. He, H.-Y. One-step assembly of $2 \mathrm{H}-1 \mathrm{~T} \mathrm{MoS}_{2}$ : Cu/reduced graphene oxide nanosheets for highly efficient hydrogen evolution. Sci. Rep. 7, 45608 (2017).

19. Zhou, X. et al. Symmetric synergy of hybrid $\mathrm{CoS}_{2}-\mathrm{WS}_{2}$ electrocatalysts for the hydrogen evolution reaction. J. Mater. Chem. A 5, 15552-15558 (2017).

20. Shifa, T. A. et al. Efficient Catalysis of Hydrogen Evolution Reaction from $\mathrm{WS}_{2(1-\mathrm{x})} \mathrm{P}_{2 \mathrm{x}}$ Nanoribbons. Small 13 (2017).

21. Geng, X. et al. Pure and stable metallic phase molybdenum disulfide nanosheets for hydrogen evolution reaction. Nat. Commun. 7 , $10672(2016)$

22. Li, H. et al. Amorphous nickel-cobalt complexes hybridized with 1T-phase molybdenum disulfide via hydrazine-induced phase transformation for water splitting. Nat. Commun. 8, 15377 (2017).

23. Leong, S. X. et al. $2 \mathrm{H} \rightarrow 1 \mathrm{~T}$ Phase Change in Direct Synthesis of $\mathrm{WS}_{2}$ Nanosheets via Solution-Based Electrochemical Exfoliation and Their Catalytic Properties. ACS Appl. Mater. Inter. 9, 26350-26356 (2017).

24. Wang, D.-Y. et al. Highly active and stable hybrid catalyst of cobalt-doped $\mathrm{FeS}_{2}$ nanosheets-carbon nanotubes for hydrogen evolution reaction. J. Am. Chem. Soc. 137, 1587-1592 (2015).

25. Lei, Z., Xu, S. \& Wu, P. Ultra-thin and porous $\mathrm{MoSe}_{2}$ nanosheets: facile preparation and enhanced electrocatalytic activity towards the hydrogen evolution reaction. Phys. Chem. Chem. Phys. 18, 70-74 (2016).

26. Wang, X. et al. Noble-Metal-Free Hybrid Membranes for Highly Efficient Hydrogen Evolution. Adv. Mater. 29 (2017).

27. Liu, F. et al. A catalyzed-growth route to directly form micropatterned $\mathrm{WO}_{2}$ and $\mathrm{WO}_{3}$ nanowire arrays with excellent field emission behaviors at low temperature. Cryst. Growth Des. 10, 5193-5199 (2010).

28. Zheng, T. et al. Conductive Tungsten Oxide Nanosheets for Highly Efficient Hydrogen Evolution. Nano Lett. 17, 7968-7973 (2017).

29. Chen, J. et al. $\mathrm{WO}_{3-\mathrm{x}}$ Nanoplates Grown on Carbon Nanofibers for an Efficient Electrocatalytic Hydrogen Evolution Reaction. ACS Appl. Mater. Inter. 8, 18132-18139 (2016).

30. Morrish, R., Haak, T. \& Wolden, C. A. Low-temperature synthesis of n-type $\mathrm{WS}_{2}$ thin films via $\mathrm{H}_{2} \mathrm{~S}$ plasma sulfurization of WO . Chem. Mater. 26, 3986-3992 (2014).

31. Huo, N. et al. Novel and Enhanced Optoelectronic Performances of Multilayer $\mathrm{MoS}_{2}-\mathrm{WS}_{2}$ Heterostructure Transistors. Adv. Funct. Mater. 24, 7025-7031, https://doi.org/10.1002/adfm.201401504 (2014). 
32. Shifa, T. A. et al. Engineering the Electronic Structure of $2 \mathrm{D} \mathrm{WS}_{2}$ Nanosheets Using Co Incorporation as $\mathrm{Co}_{\mathrm{x}} \mathrm{W}_{(1-\mathrm{x})} \mathrm{S}_{2}$ for Conspicuously Enhanced Hydrogen Generation. Small 12, 3802-3809, https://doi.org/10.1002/smll.201601168 (2016).

33. Zhang, J. et al. Copper dopants improved the hydrogen evolution activity of earth-abundant cobalt pyrite catalysts by activating the electrocatalytically inert sulfur sites. J. Mater. Chem. A 5, 17601-17608, https://doi.org/10.1039/c7ta05433e (2017).

34. Li, Y. et al. $\mathrm{MoS}_{2}$ Nanoparticles Grown on Graphene: An Advanced Catalyst for the Hydrogen Evolution Reaction. J. Am. Chem. Soc. 133, 7296-7299, https://doi.org/10.1021/ja201269b (2011).

35. Drisko, G. L. et al. Air-Stable Anisotropic Monocrystalline Nickel Nanowires Characterized Using Electron Holography. Nano Lett., https://doi.org/10.1021/acs.nanolett.7b04791 (2018)

36. Li, L., Hu, X. \& Gao, Y. Electron Holographic Study of Semiconductor Light-Emitting Diodes. Small 14, https://doi.org/10.1002/ smll.201701996 (2018).

37. Cao, Y. et al. Atomic-Level Insight into Optimizing the Hydrogen Evolution Pathway over a Co-1-N-4 Single-Site Photocatalyst. Angew. Chem. Int. Ed. 56, 12191-12196, https://doi.org/10.1002/anie.201706467 (2017).

\section{Acknowledgements}

This work was financially supported by the National Basic Research Program of China (2018YFA0209100), the National Science Foundations of China (61205057 and 11574136).

\section{Author Contributions}

The experiments and characterizations were carried out by L.Y.Z. with the assistence of H.Z.S. and H.W. under the guidance of S.C.Y. and Y.S. L.Y.Z. and S.C.Y. wrote the manuscript and prepared all figures. Y.S. and S.C.Y. supervised and coordinated all the work.

\section{Additional Information}

Supplementary information accompanies this paper at https://doi.org/10.1038/s41598-018-37598-0.

Competing Interests: The authors declare no competing interests.

Publisher's note: Springer Nature remains neutral with regard to jurisdictional claims in published maps and institutional affiliations.

Open Access This article is licensed under a Creative Commons Attribution 4.0 International License, which permits use, sharing, adaptation, distribution and reproduction in any medium or format, as long as you give appropriate credit to the original author(s) and the source, provide a link to the Creative Commons license, and indicate if changes were made. The images or other third party material in this article are included in the article's Creative Commons license, unless indicated otherwise in a credit line to the material. If material is not included in the article's Creative Commons license and your intended use is not permitted by statutory regulation or exceeds the permitted use, you will need to obtain permission directly from the copyright holder. To view a copy of this license, visit http://creativecommons.org/licenses/by/4.0/.

(c) The Author(s) 2019 